\section{REVIEW ARTICLE}

\title{
Endless possibilities: translation termination and stop codon recognition
}

\author{
Gwyneth Bertram, $\uparrow$ Shona Innes, $\uparrow$ Odile Minella, $†$ \\ Jonathan P. Richardson, $†$ and Ian Stansfield
}

Author for correspondence: Ian Stansfield. Tel: +44 1224 273106. Fax: +44 1224273144. e-mail: i.stansfield@abdn.ac.uk

Department of Molecular and Cell Biology, University of Aberdeen, Institute of Medical Sciences, Foresterhill, Aberdeen AB25 2ZD, UK

Keywords: translation termination, protein synthesis, stop codon, release factor, nonsense suppression

\section{Overview}

The process of protein synthesis can be divided into three main phases: initiation, during which the ribosomal subunits join the mRNA and locate the AUG initiator codon; elongation, during which sense codons are decoded and the bulk of the polypeptide is made; and termination, during which a stop codon directs the release of the completed polypeptide from the ribosome. Whereas the general principles of sense codon decoding by transfer RNAs are well established, a clear picture of translation termination and stop codon recognition has hitherto been lacking. Recently however, the use of optimized, complex, reconstituted in vitro termination reactions to identify the roles of key termination factors, and the solution of tertiary structures of termination factors, has allowed a reappraisal of termination factor structure and function. This review will describe these recent advances, many of which have resulted from studies of termination in micro-organisms, and in the light of the new information, discuss models for the mechanism of termination and recognition of the stop codon. While the mechanism of peptide chain termination is normally effective, stop codons are not always recognized efficiently, and during the translation of certain viral RNAs can be suppressed or read through, resulting in the expression of additional coding information. How stop codons are reassigned to encode 'sense' at a given frequency in some RNAs will be reviewed in the context of current understanding of termination and stop codon recognition mechanisms.

\section{The translation termination apparatus in eukaryotes and prokaryotes}

During translation termination, a stop codon located in the ribosomal A-site is recognized by a release factor or

†These authors contributed equally to the work. release factor complex, which binds the ribosome and triggers release of the nascent peptide. In eukaryotes, translation is terminated by a heterodimer consisting of two proteins, release factors eRF1 and eRF3, which interact in vivo (Frolova et al., 1994; Zhouravleva et al., 1995; Stansfield et al., 1995). eRF1 recognizes all three stop codons and triggers peptidyl-tRNA hydrolysis by the ribosome, releasing the nascent peptide (Frolova et al., 1994; Drugeon et al., 1997). Eukaryote termination efficiency is enhanced by the GTPase release factor eRF3, the second component of the heterodimer eRF complex. In response to a stop codon in the ribosomal A-site, formation of a quaternary complex comprising the ribosome, eRF1, GTP and eRF3 triggers GTP hydrolysis and enhances the rate of peptidyl release (Zhouravleva et al., 1995 ; Frolova et al., 1994; Fig. 1). In yeast, eRF1 and eRF3 are encoded by essential genes (SUP35 and SUP45, respectively), mutations in which produce nonsense suppression phenotypes (Stansfield \& Tuite, 1994).

In contrast to eukaryotes, the role of stop codon recognition during translation termination in eubacteria is divided between two so-called class 1 release factors, RF1 and RF2, which in Escherichia coli are encoded by the essential prfA and $\operatorname{prfB}$ genes, respectively (Scolnick et al., 1968; Caskey et al., 1984; Weiss et al., 1984). RF1 catalyses translation termination at UAA and UAG codons, and RF2 at UAA and UGA codons (Craigen et al., 1990; Fig. 1). Since RF2 bound to the ribosomal Asite can be chemically cross-linked to an artificial mRNA containing a UAA stop codon, this recognition must be achieved through direct physical contact (Lang et al., 1989; Brown \& Tate, 1994). The mRNA bases following the stop codon can also be cross-linked to RF2, implying the release factor has a footprint broader than a single codon span (Poole et al., 1998). Consistent with this, $3^{\prime}$ nucleotide stop codon context has a marked influence on the efficiency of stop codon recognition in bacteria, yeast and human cells (Poole et al., 1995; Bonetti et al., 

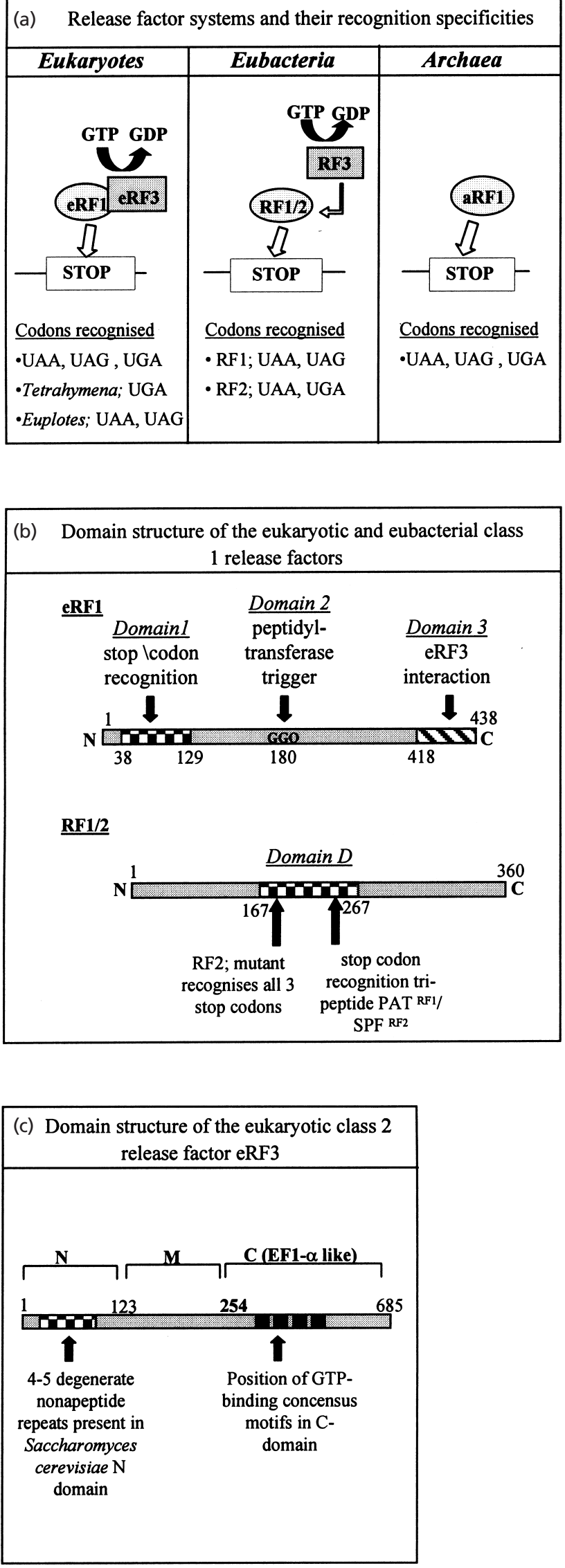

1995; McCaughan et al., 1995). In prokaryotes, dissociation of RF1 and RF2 from the ribosome is stimulated by the addition of a GTPase release factor, RF3, analogous to eukaryote eRF3 (Freistroffer et al., 1997). However, unlike eRF3, RF3 is non-essential and does not form stable complexes with RF1 and RF2, implying these latter RFs can function in the absence of RF3 (Grentzmann et al., 1994; Mikuni et al., 1994).

\section{The role of GTPase release factors in termination}

eRF3 and RF3 show limited sequence similarity to one another at the amino acid level, and while the eRF3 Cterminal domain shows most sequence similarity to eukaryotic elongation factor EF- $1 \alpha$ (which brings aminoacyl tRNAs to the A-site), RF3 is most similar to prokaryotic elongation factor EF-G (the ribosome translocase), implying their precise functions may differ. A study of in vitro termination reactions has shown that in single turnover reactions, peptidyl release is independent of RF3, and that the rate of RF1/2 association with the ribosome is independent of RF3. This evidence supports a model in which RF3 may stimulate termination efficiency by ejecting the class 1 release factors RF1/2 from the A-site following peptidyl-tRNA hydrolysis (Freistroffer et al., 1997). RF3 also enhances the efficiency of RF2-mediated termination at less preferred (UGA) stop codons, or at stop codons in weak contexts (Crawford et al., 1999; Grentzmann et al., 1995). However, there is as yet no evidence that eRF3 functions in a similar way to eject eRF1 from the A-site following termination. Instead, some observations suggest that eRF1 and eRF3 might act as a complex to mediate peptidyl release; in yeast and mammals, eRF1 and eRF3 form a complex in vivo and in vitro (Zhouravleva et al., 1995), and in yeast, overexpression of both eRF3 and eRF1 together is required to cause large increases in translation termination efficiency and thereby outcompete suppressor tRNAs for stop codon binding (Stansfield et al., 1995). However, the demonstration that eRF1 alone has peptidyl-release activity in vitro argues that, at least in a reconstituted system, an (EF1$\alpha$-like) eRF3 system to deliver eRF1 to the A-site is not a

Fig. 1. Release factors and their domain structures. (a) Release factor complexes in eukaryotes, eubacteria and archaea. Some eukaryote genera, such as Tetrahymena and Paramecium (UGA signals stop) and Euplotes (UAR signals stop) use a restricted set of stop codons. This may result from an altered stop codon recognition ability of the eRF1s in these organisms. (b) Domain structure of class 1 release factors from eukaryotes and eubacteria, showing the functionally important domains (see text for detailed discussion). (c) Domain structure of the eukaryote class 2 release factors. The three assigned N, M and C domains are shown, as are the approximate position of the nonapeptide repeat motifs present in the $S$. cerevisiae eRF3 sequence which are required for inheritance of the $\left[\mathrm{PSI}^{+}\right]$ prion state (see text for details). Figures next to rectangles representing the release factor polypeptides denote amino acid numberings. 
requirement (Frolova et al., 1994). Secondly, in mammalian cells and in vitro systems, overexpression of eRF1 alone is sufficient to outcompete suppressor tRNAs for stop codon binding (Legoff et al., 1997). Recent results have also cast doubt on the early models suggesting that eRF1 and eRF3 interaction is a requirement for function; the in vivo interaction of yeast eRF1 and eRF3 is mediated by the C-terminal-most 6-11 amino acids of eRF1 (Eurwilaichitr $\mathrm{et}$ al., 1999; Ito $\mathrm{et}$ al., 1998a). However, it is possible to trim off the yeast eRF1 $\mathrm{C}$-terminal residues to a point where eRF3 interaction is no longer detectable, and yet still maintain yeast viability, although stop codon recognition is somewhat impaired, producing a nonsense suppression phenotype (Eurwilaichitr et al., 1999; Ito et al., 1998a). It may therefore be unnecessary to form an eRF1-eRF3 complex in order to terminate translation, although it remains possible that eRF3 may still have affinity for a ribosome-eRF1-stop codon complex. Subsequently, eRF3 could perform a central (essential) role in termination, either enhancing peptidyl release, or perhaps recycling eRF1 from post-peptidyl-release ribosomes in an RF3-like manner. Such models are speculative however, and much more detailed biochemical evidence is required before the mechanism of eukaryote termination can be elucidated. Interestingly, archaea also have eukaryote-like eRF1s (aRF1), although intriguingly, without exception they lack the eRF1 Cterminal amino acids which in yeast interact with eRF3 (Inagaki \& Doolittle, 2000). However, it is not clear that archaea even have an eRF3 counterpart, although for instance Archeoglobus fulgidus possesses two EF1 $\alpha / \mathrm{EF}-$ $\mathrm{Tu}$-like proteins, one of which could conceivably have class 2 release factor function. These observations support the hypothesis that eRF1-eRF3 interaction may not be required for the stop codon recognition and termination reactions (Fig. 1).

In mouse, two eRF3 variants have been identified which differ in their $\mathrm{N}$-terminal domain sequences and expression patterns in this organism (Hoshino et al., 1998). However, whether or not they have distinct activities in termination remains to be addressed. Intriguingly, one mouse eRF3 is known to interact with the $\mathrm{C}$ terminus of mammalian poly(A)-binding protein PABP (Hoshino et al., 1999), raising a number of questions about the breadth of potential functions this protein may carry out, including involvement in the regulation of mRNA stability and, potentially, recycling post-termination ribosomes back to the $5^{\prime}$ end of the mRNA to participate in new rounds of translation initiation (Hoshino et al., 1999).

\section{RF recognition of the stop codon}

One important, as yet unanswered question concerns the mechanism of stop codon recognition by the release factor. The ability to cross-link RF2 to a stop codon indicates that the class 1 release factors may exhibit a tRNA-like activity (Poole et al., 1998). One hypothesis is that release factors may mimic a tRNA both structurally and functionally. This model was prompted by the discovery by Nissen and colleagues that the structure of elongation factor EF-G (GDP) from Thermus thermophilus resembles that of a tRNA-EF-Tu-GTP complex, with EF-G domain IV mimicking major portions of a tRNA, including the T-stem and anticodon loop (Fig. 2; Nissen et al., 1995; Ito et al., 1996). This hypothesis was developed by Ito and co-workers, who suggested that prokaryotic class 1 release factors, which share weak sequence homology across their central D and $\mathrm{E}$ domains to EF-G domain IV, may also 'mimic' a tRNA molecule. $\operatorname{prfB}$ (RF2) mutants were subsequently isolated which could complement conditional-lethal RF1 and RF2 mutations, i.e. an RF2 molecule capable of recognizing all three stop codons; the mutation (E167K) mapped within the central D domain of RF2 (Ito et al., 1998b). More recently, elegant experiments swapping domains between RF1 and RF2 have exchanged stop codon recognition specificity between the two prokaryote RFs. In this way, an RF domain responsible for stop codon specificity was subsequently narrowed down to a single tripeptide (RF1, Pro Ala Thr; RF2, Ser Pro Phe), again in the central domain D, where the weak similarity to EFG domain IV was located (Ito et al., 2000). However, final confirmation that this tripeptide represents the actual site of stop codon interaction will require chemical cross-linking data (stop codon-RF), or cocrystallization of the stop codon and RF.

The RF-tRNA mimicry proposal is also supported by the recent determination of the crystal structure of eukaryote eRF1 (human) revealing a Y-shaped molecule with similar dimensions to a tRNA (Song et al., 2000; Fig. 2). The eRF1 GGQ motif, which triggers peptidyl release once the release factor binds to the stop codon (Frolova et al., 1999), is located in one of the arms of the $\mathrm{Y}$, in domain 2. Domain 2 lies $80 \AA$ from eRF1 domain 1 (comprising the $\mathrm{N}$-terminal third of the protein) in the stem of the Y (Fig. 2). This distance is very similar to the $75 \AA$ which separates the CCA acceptor stem of a tRNA molecule (which triggers peptidyl transferase during elongation) and the tRNA anticodon. eRF1 domain 1 is thus a likely candidate to represent the anticodon-like structural element in the release factor (Song et al., 2000). Consistent with this, a series of mutations have recently been identified in yeast eRF1 domain 1 which alter the stop codon recognition specificity of the release factor, and which define pockets potentially capable of binding stop codon trinucleotides (Bertram et al., 2000). These studies, and the tRNA-like shape of eRF1, provide evidence that this domain could play some role in recognizing the stop codon. The $\mathrm{N}$-terminal location of this putative anticodon-like region is dissimilar to the central location of the discriminator tripeptide in RF1/2 (Ito et al., 2000), inferring eukaryote and prokaryote class 1 release factors may have very dissimilar protein folds, although both may have an overall tRNA-like shape. Again, confirmation of models for eukaryote eRF1-stop codon interaction requires stop codon/eRF1 co-crystallization or cross-linking data, and the functional relevance of the putative eRF1-tRNA structural similarity remains to be established. 
(a) EF-Tu-GTP-tRNA

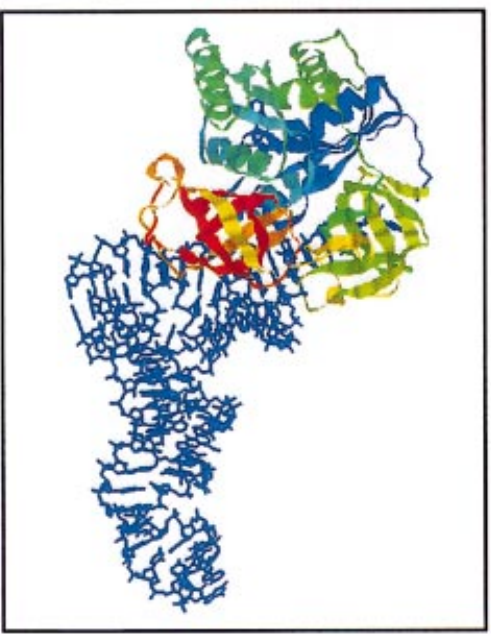

(c) tRNA Phe (b) EF-G-GDP

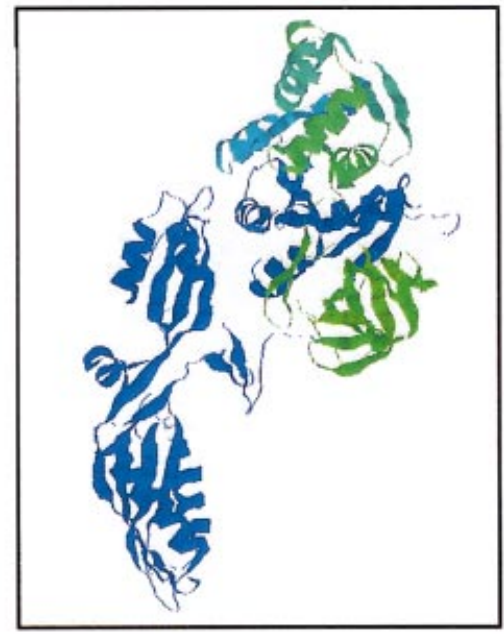

(d) eRF1

(e) RRF

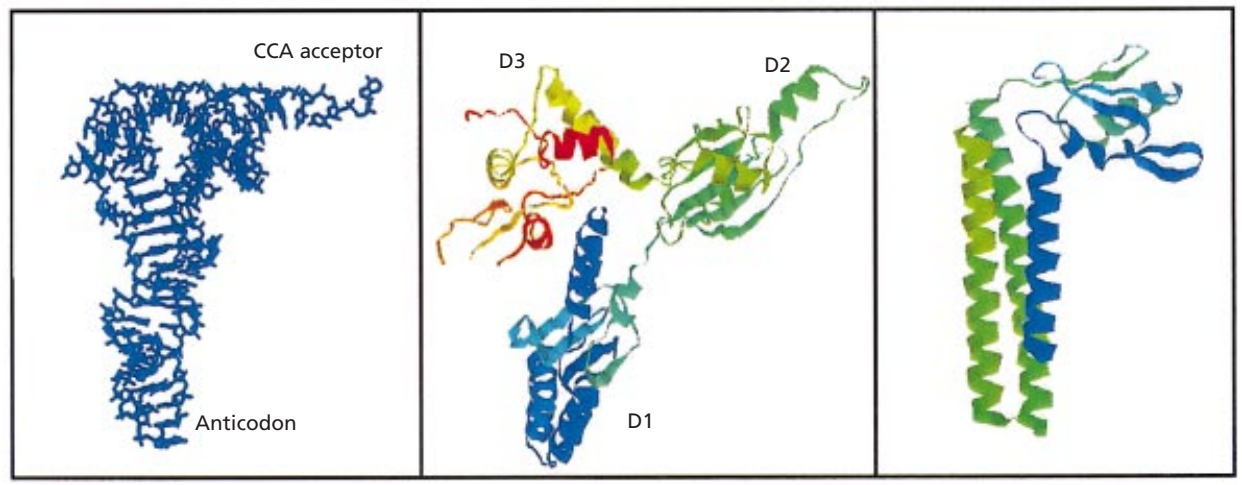

Fig. 2. Molecular mimicry amongst translation elongation and termination factors from prokaryotes and eukaryotes. (a) EF-Tu-tRNA-GTP complex (Nissen et al., 1995), with tRNA in stick format coloured blue and protein in ribbon format. (b) EF-G in the GDP-bound state (Al Karadaghi et al., 1996), with domains III, IV and V acting as a tRNA mimicry structure and coloured blue. (c) Crystal structure of yeast tRNA ${ }^{\text {Phe, }}$, coloured blue (Robertus et al., 1974). The anticodon and CCA acceptor are labelled. (d) Crystal structure of human eRF1 in ribbon format, aligned to show tRNA-like overall shape and dimensions (Song et al., 2000). The three domains referred to in the text and in Fig. 1 are labelled D1-D3. (e) Secondary structure ribbon diagram of the crystal structure of Thermus thermatoga ribosome recycling factor (RRF; Selmer et al., 1999) aligned to show the tRNA-like shape and dimensions. Except where stated, molecule chains are coloured using a continuous spectrum starting at blue ( $\mathrm{N}$ terminus), passing through green, yellow and orange, to red at the $\mathrm{C}$ terminus.

\section{Release factors and the reassignment of stop codons to sense}

Deviations from the universal code have been identified in both the mitochondrial and the nuclear genomes of various organisms. In the prokaryotes Mycoplasma genitalium, Mycoplasma capricolum, Mycoplasma pneumoniae and Mycoplasma gallisepticum, the UGA codon is not used for termination but instead encodes tryptophan (Inamine et al., 1990; Yamao et al., 1985) due to the presence of a tRNA $\mathrm{ACU}_{\mathrm{H}}$, which translates both UGA and UGG codons. While the genome of M. genitalium contains a prfA gene encoding RF1, there is no homologue of the gene encoding UGA-decoding RF2 (prfB; Fraser et al., 1995).

Amongst eukaryote micro-organisms, the ciliate species of the genera Paramecium and Tetrahymena use UAA and UAG to encode glutamine (Caron \& Meyer, 1985), while Euplotes species signal stop using UAA and UAG only, with UGA encoding cysteine (Meyer et al., 1991). Recently, an unclassified diplomonad species from the Hexamitidae has been discovered with an apparent stop codon reassignment to glutamine (Keeling \& Doolittle, 1997). These discoveries have led to the search for the corresponding tRNAs which decode the stop codons as sense. In Tetrahymena, a tRNA $\mathrm{CUA}_{\mathrm{CU}}^{\mathrm{Gln}}$ and a tRNA $\mathrm{UmUA}_{\mathrm{Um}}^{\mathrm{Gln}}$ (where Um represents 2'-O-methyluridine) have anticodons cognate for UAG and UAA, respectively (Hanyu et al., 1986; Kuchino et al., 1985; Table 1). A third tRNA $\mathrm{UmUG}_{\mathrm{Um}}^{\mathrm{Gln}}$ decodes both the glutamine codons CAA and CAG. The diplomonads with reassigned stop codons use similar tRNA Gln and tRNA $\mathrm{CU}_{\mathrm{UUA}}^{\mathrm{Gln}}$ (Keeling \& Doolittle, 1997).

In contrast to these stop codon cognate tRNAs, a Euplotes cysteinyl-tRNA decoding UGA has the non- 
Translation termination and stop codon recognition

Table 1. Properties of example stop-codon-decoding and natural suppressor tRNAs from different species

\begin{tabular}{|c|c|c|c|c|c|c|}
\hline & tRNA & Species & $\begin{array}{l}\text { Anticodon } \\
\left(5^{\prime}-3^{\prime}\right)\end{array}$ & $\begin{array}{l}\text { Codon }(s) \\
\text { naturally } \\
\text { decoded }\end{array}$ & $\begin{array}{l}\text { Stop codon } \\
\text { decoded }\end{array}$ & $\begin{array}{l}\text { Conditions } \\
\text { required for stop } \\
\text { codon decoding* }\end{array}$ \\
\hline \multicolumn{7}{|c|}{ Stop codon cognate tRNAs from non-universal code species } \\
\hline & tRNA ${ }^{\text {Gln }}$ & $\begin{array}{l}\text { Tetrabymena } \\
\text { thermophila }\end{array}$ & UmUA & UAA & UAA & - \\
\hline & $\mathrm{tRNA}^{\mathrm{Gln}}$ & $\begin{array}{r}\text { Tetrabymena } \\
\text { thermophila }\end{array}$ & CUA & UAG & UAG & - \\
\hline & tRNA ${ }^{\mathrm{Cys}}$ & $\begin{array}{l}\text { Euplotes } \\
\text { octocarinatus }\end{array}$ & GCA & UGG, UGA & UGA & - \\
\hline \multicolumn{7}{|c|}{ Natural suppressor tRNAs from universal genetic code species } \\
\hline \multirow[t]{3}{*}{ UAA, UAG } & $\mathrm{tRNA}^{\mathrm{Gln}}$ & $\begin{array}{l}\text { Saccharomyces } \\
\text { cerevisiae }\end{array}$ & UUG & CAA & UAA & Overexpression \\
\hline & $\mathrm{tRNA}^{\mathrm{G} l \mathrm{n}}$ & $\begin{array}{l}\text { Saccharomyces } \\
\text { cerevisiae }\end{array}$ & CUG & CAG & UAG & Overexpression \\
\hline & $\mathrm{tRNA}^{\mathrm{Tyr}}$ & Nicotiana rustica & 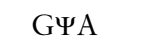 & UGG & UAA, UAG & TMV leaky stop context \\
\hline \multirow[t]{3}{*}{ UGA } & $\mathrm{tRNA}^{\mathrm{Cys}}$ & Nicotiana rustica & GCA & UGC & UGA & TMV leaky stop context \\
\hline & tRNA ${ }^{\operatorname{Arg}}$ & Wheat & $\mathrm{U}^{*} \mathrm{CG}$ & CGA & UGA & $\begin{array}{l}\text { TRV/PEMV leaky stop } \\
\text { context }\end{array}$ \\
\hline & tRNA ${ }^{\operatorname{Trp}}$ & Nicotiana rustica & $\mathrm{CmCA}$ & UGG & UGA & $\begin{array}{l}\text { TRV/TMV/PEMV leaky } \\
\text { stop context }\end{array}$ \\
\hline
\end{tabular}

* TMV, tobacco mosaic virus; TRV, tobacco rattle virus; PEMV, pea enation mosaic virus. References are given in the text.

UGA cognate anticodon GCA, requiring a G:A mispairing event at the third codon position. It appears to be the only $\mathrm{tRNA}^{\mathrm{Cys}}$ in the Euplotes genome, also decoding the canonical cysteine codon UGC (Grimm et al., 1998). While this is not an obvious solution to the UGA decoding problem, a plant tRNA $\mathrm{GCA}_{\mathrm{GCA}}^{\mathrm{Cys}}$ is known with UGA suppressor activity, although this is only efficient where the UGA codon is placed in a poor nucleotide context (Urban \& Beier, 1995). It is not clear whether to achieve efficient UGA decoding, Euplotes employs an altered release factor disabled for UGA recognition, or a ciliate tRNA ${ }^{\mathrm{Cys}}$ with unique decoding properties, or both. However, the Euplotes tRNA ${ }^{\text {Cys }}$ does not appear to be unusual structurally when compared to other known tRNA ${ }^{\mathrm{Cys}}$, and UGA sense codons in Euplotes genes are not found in nucleotide contexts unfavourable for eRF1 binding (see below), suggesting eRF1 altered stop specificity may be an important component of codon reassignment (Grimm et al., 1998). It is also feasible that a UGA-cognate Euplotes tRNA still remains to be discovered. Recently, the eRF1 gene sequence from Tetrahymena thermophila has been isolated; it encodes an eRF1 protein highly homologous at the amino acid level to other eukaryotic and archaeal release factors (e.g. $57 \%$ identity with human eRF1), but it is unclear which, if any, amino acid substitutions are responsible for altered stop codon specificity (Karamyshev et al., 1999). However, it is known that the Tetrabymena eRF1 is unable to complement a yeast temperature-sensitive mutation in the SUP45 gene (encoding eRF1), consistent, among other possibilities, with a restricted stop codon recognition specificity (Karamyshev et al., 1999).

\section{The termination signal: stop codons and the role of flanking nucleotides}

It is well established that not only is the relative usage of the three types of stop codon biased, but also the nucleotide contexts in which they are found is nonrandom, particularly for those genes that are highly expressed. The identity of the base directly following the termination codon (the +1 base) is subject to particular bias, but nucleotides as far downstream as nucleotide position +3 are also non-random; experiments measuring competition between termination and other non-standard translational events, such as frameshifting, nonsense suppression or selenocysteine incorporation, reveal that the base in this position affects termination efficiency in vivo in bacteria, yeast and humans, respectively (Major et al., 1996; Bonetti et al., 1995; McCaughan et al., 1995). The stop codon then has characteristics of a tetranucleotide, where the identity of the fourth base determines the efficiency with which termination occurs. Contexts favouring termination are predominantly used by highly expressed genes in all micro-organisms so far examined (Bonetti et al., 1995; Poole et al., 1995). Conversely, viral genomes containing stop codons whose suppression is required to direct translation of extended proteins have stop codons in poor contexts, favouring suppression. The tetranucleotide stop codon hypothesis has been strengthened considerably by studies showing that bacterial release factor RF-2 can be directly cross-linked to the nucleotide in the +4 position, as well as nucleotides further downstream, supporting the idea that as many as three succeeding nucleotides may contact the release factor 
and have roles as termination efficiency determinants (Poole et al., 1998).

The $5^{\prime}$ nucleotide context of a stop codon also influences how efficiently a stop codon directs termination. In $E$. coli, this operates partly through the identity of the penultimate amino acid residue in the nascent peptide, which influences termination efficiency by up to 30 -fold (Mottagui-Tabar et al., 1994). Efficient termination events are associated with penultimate amino acid residues that are basic in nature. The nature of the Cterminal (ultimate) amino acid residue, whose codon is located immediately $5^{\prime}$ to the termination signal, also modulates termination efficiency in E. coli (Bjornsson et al., 1996). Termination efficiency is stimulated synergistically by pairings of the last two (ultimate and penultimate) amino acids of a polypeptide chain, which increase the propensity of $\alpha$-helix or $\beta$-sheet formation, pairings which are represented most frequently in highly expressed genes in both E. coli and Bacillus subtilis (Bjornsson et al., 1996; Mottagui-Tabar \& Isaksson, 1998). The $5^{\prime}$ codon context effect in E. coli is also partly mediated by the identity of the tRNA isoacceptor in the ribosomal P-site (Bjornsson et al., 1996). In Saccharomyces cerevisiae, termination by eRF1 is less influenced by the penultimate amino acid in the peptide chain; the greater effect is exerted by the P-site tRNAs. While a Psite CAG-decoding glutamine tRNA is not particularly antagonistic to release factor binding, CAA-decoding tRNA Gln , when in the P-site, increases suppression of an A-site stop codon far more (Mottagui-Tabar et al., 1998). These effects almost certainly occur through stearic interactions between the P-site tRNA and the release factor (Bjornsson et al., 1996). Context effects thus have profound influences on termination efficiency in both prokaryotic and eukaryotic systems, and stop codon contexts which are poor substrates for eRF1 recognition are employed by viruses to direct stop codon suppression, as will be discussed below.

\section{Programmed stop codon readthrough}

While in organisms using the standard genetic code efficient recognition of the three stop codons is required for viability, in some viral genomes and cellular genes readthrough of stop codons is an integral part of the control of gene expression. Thus at a certain frequency, alternative translation elongation events take place instead of the default termination process. Regulation of this type controls the ratio of correctly terminated translation product to that of the polypeptide generated by stop codon readthrough. The readthrough of inframe stop codons is driven by various cis-acting signals, almost always including a stop codon context which is suboptimal for release factor recognition, but which in some cases can include readthrough-enhancing RNA secondary structure elements downstream of the stop codon. How such secondary structures downstream of the stop codon can negatively influence successful eRF decoding is at present unknown, but clearly elements comparatively remote from the stop codon can have important effects on eRF-codon recognition.

\section{Viral and retrotransposon stop codon suppression}

Translation of tobacco mosaic virus (TMV) main genomic RNA results in the synthesis of two polypeptides of $126 \mathrm{kDa}$ and $183 \mathrm{kDa}$ with a molar ratio of $20: 1$. Synthesis of the larger polypeptide results from the translational readthrough of a UAG stop codon, possibly by a tRNA ${ }_{\mathrm{AUG}}^{\mathrm{Tyr}}$ which has known in vitro suppression activity (Pelham, 1978; Beier et al., 1984). This readthrough process is essential for viability of the virus and controls the level of RNA replicase (Ishikawa et al., 1986). The five nucleotides following the stop codon with the consensus sequence UAG CAR YYA, including the cytosine nucleotide immediately $3^{\prime}$ to the UAG stop codon, play an important role in readthrough of the TMV stop coding signal, stimulating 2-5\% suppression (Skuzeski \& Atkins, 1990; Goelet et al., 1982; Skuzeski et al., 1991; Table 2). Since no RNA secondary structural elements have been identified in the leaky stop codon environment (Goelet et al., 1982), it seems likely that the $3^{\prime}$ stop codon context alone directs readthrough (Skuzeski et al., 1991). Programmed readthrough of the $\mathrm{Q} \beta$ bacteriophage coat protein gene UGA codon by a tRNA ${ }^{\mathrm{Trp}}$ is also driven by nucleotide context 3' of the stop codon (Weiner \& Weber, 1973; EngelbergKulka, 1981; Table 2).

Table 2. Regulatory recoding signals directing the sense translation of viral and cellular gene stop codons

See text for details. ND, Not determined.

\begin{tabular}{|c|c|c|c|c|c|c|}
\hline System & $\begin{array}{l}\text { Secondary } \\
\text { structure }\end{array}$ & $5^{\prime}$ codon & Stop codon & $3^{\prime}$ codon & Spacer region size & Other sequence requirements \\
\hline $\begin{array}{l}\text { Moloney murine leukaemia virus } \\
\text { (Mo-MuLV) }\end{array}$ & Pseudoknot & GAC & UAG & GGA & $8 \mathrm{nt}$ (purine-rich) & $\begin{array}{l}\text { Possible involvement of stem-loop structure which potentially } \\
\text { competes with pseudoknot for formation }\end{array}$ \\
\hline Tobacco mosaic virus (TMV) & None found & CAA & UAG & CAA & $\mathrm{ND}$ & The nucleotide $3^{\prime}$ of the UGA is very important to readthrough \\
\hline $\begin{array}{l}\text { Barley yellow dwarf virus } \\
\text { (BYDV) }\end{array}$ & ND & AAA & UAG & GUA & ND & $\begin{array}{l}\text { CN AAA UAG GUA GAC } \\
\text { Stop codon environment found in all luteoviruses; additional } \\
\text { CCN repeat region required, plus downstream sequences. } \\
\text { For additional sequences see text }\end{array}$ \\
\hline $\begin{array}{l}\text { pCal (Candida albicans } \\
\text { retrotransposon) }\end{array}$ & $\begin{array}{l}\text { Potential } \\
\text { pseudoknot }\end{array}$ & GAA & UGA & AAA & $8 \mathrm{nt}$ (purine-rich) & Potential pseudoknot? \\
\hline $\begin{array}{l}\text { Skipper (Dictyostelium } \\
\text { retrotransposon) }\end{array}$ & $\begin{array}{l}\text { Potential } \\
\text { pseudoknot }\end{array}$ & $\mathrm{CAA}$ & UGA & CUC & $\begin{array}{l}\text { Proposed to have no spacer } \\
\text { as UGA forms part of } \\
\text { stem I }\end{array}$ & Potential pseudoknot? \\
\hline
\end{tabular}


Barley yellow dwarf virus (BYDV) belongs to the luteovirus group, which use a variety of translational control mechanisms for gene expression. The BYDV coat protein (CP) ORF is separated from a second reading frame, RT, by a stop codon, whose suppression is required to form a fusion protein (Dinesh Kumar et al., 1992). The CP stop codon environment is conserved between different luteoviruses, although unique among stop codon readthrough signals (see Table 2). A 6-15 base spacer separates the stop codon and a downstream block of 7-16 tandem proline-encoding repeats. This, together with a sequence 700 bases downstream from the stop codon, drives CP-stop codon readthrough (Brown et al., 1996; Guilley et al., 1994). The stop codon distal nature of this cis sequence is unusual, although it is well conserved between the luteoviruses and functions at different distances from the stop codon.

Not all stop codon readthrough signals are solely dependent on primary sequence; some also have an RNA structural component (see Fig. 3). Moloney murine leukaemia virus (Mo-MuLV) is a well-studied example of a mammalian type $C$ retrovirus that uses UAG stop codon readthrough, with glutamine insertion at the stop codon position to express the gag and gag-pol fusion proteins in the $5 \%$ ratio required for virion production (Yoshinaka et al., 1985). Cis-acting factors directing nonsense suppression include the sequence downstream of the stop codon, which has the ability to form an RNA pseudoknot structure with a minimal sequence requirement of $60 \mathrm{nt}$ (Wills et al., 1991; Feng et al., 1992). While RNA pseudoknots are common cis signals which promote programmed ribosomal frameshifting in viral genomes (Brierley et al., 1989), they cannot be regarded as generic RNA secondary structures which can trigger any recoding event; the mouse mammary tumour virus (MMTV) pseudoknot, found downstream of the MMTV frameshift site, while similar to the Mo-MuLV pseudoknot, is unable to functionally substitute for the Mo-MuLV structure (Gesteland \& Atkins, 1996).

Retrotransposons have two long ORFs homologous to the gag and pol ORFs of retroviruses, and the yeast retrotransposon Ty1 employs ribosomal frameshifting at the gag-pol junction to regulate gag:gag-pol translation products (Clare et al., 1988). However, in the case of both the Candida albicans retrotransposon pCal and the Dictyostelium discoideum retrotransposon skipper, gag and pol ORFs are in the same reading frame, separated by a UGA termination codon (Matthews et al., 1997; Leng et al., 1998), a novel retrotransposon coding arrangement. In both cases, the UGA stop codon separating gag and pol is followed by a potential pseudoknot-forming sequence, similar to the Mo-MuLV readthrough signal, an indication that readthrough of the UGA stop codon may be programmed and represent a common solution to the problem of regulating retrotransposon gene expression (Matthews et al., 1997; Leng et al., 1998).

The mechanism by which an RNA pseudoknot can trigger stop codon readthrough or ribosomal frame- shifting is unknown. Evidence from initiation-synchronized in vitro translation reactions suggests that pseudoknots pause ribosomes at the infectious bronchitis virus (IBV) frameshift site, although not all RNA secondary structures which cause pausing are sufficient to trigger frameshifting (Somogyi et al., 1993). During programmed stop codon readthrough, however, pseudoknots must perform a different overall function to that in stop codon readthrough, although there may still be a role for pseudoknots in pausing the ribosome. Clearly, by definition, readthrough of a stop codon implies a tRNA-mediated translation elongation event at the expense of release factor recognition of the stop codon. Interaction of the pseudoknot with the ribosome may favour tRNA interaction over eRF1 binding at the Asite, perhaps by distorting the mRNA structure, or constraining its flexibility. This might in turn restrict the necessary 'presentation' of a stop codon to the release factor for recognition. Such presentation effects could operate synergistically with the unfavourable nucleotide context of the viral stop codon being read through to reduce the efficiency of eRF1-stop codon recognition.

\section{Programmed stop codon readthrough in cellular genes}

CFA/II strains of enterotoxigenic E. coli (ETEC) express three types of surface-associated hair-like fimbriae (coli surface antigens) known as CS1, CS2 and CS3. Expression of the genes required for biosynthesis and assembly of CS3 pili requires the suppression of an amber stop codon to produce a $104 \mathrm{kDa}$ protein (Jalajakumari et al., 1989). While CFA/II strains have a nonsense suppressor mutant tRNA ${ }^{\mathrm{Gln}}(\sup E)$, it is believed that in a wild-type tRNA background, the extended protein is still produced although its reduced abundance does not allow pilus synthesis (Jalajakumari et al., 1989). The amino acid (glutamine) inserted by suppression at the stop codon is specifically required for protein activity, since when tyrosine is inserted by the supF tRNA ${ }^{\mathrm{Tyr}}$, protein function is lost.

During translation in a range of species studied, UGA codons in cellular mRNAs can direct the insertion of a twenty-first amino acid, selenocysteine. This recoding event occurs in response to a specific set of trans factors and cis signals, which differ between eukaryotes and prokaryotes. Prokaryotes direct selenocysteine insertion at specific UGA codons using a 40 nt structured RNA sequence or SECIS element (Bock et al., 1991). The E. coli SelB protein, a homologue of the translation elongation factor EF-Tu, binds to the SECIS element using its C-terminal domain, bringing a UGA-decoding selenocysteine tRNA $^{\text {Sec }}$ to the UGA codon at the ribosomal A-site (Bock et al., 1991; Kromayer et al., 1996). In eukaryote systems, the SECIS element is located in the $3^{\prime}$ untranslated region (UTR) of the mRNA encoding the selenoprotein, distal to the UGA codon directing selenocysteine incorporation, and the trans factors are less well characterized (Berry et al., 1993, 1991). Obviously, the key to selenocysteine UGA 
recoding is the elimination of release factor termination at the UGA selenocysteine signal. In eukaryote systems, this is in part achieved by the poor nucleotide context in which the selenocysteine UGA codons are found. Improving this context reduces selenocysteine incorporation at the expense of termination (McCaughan et al., 1995). However, readthough efficiencies of $4-5 \%$ at the E. coli fdhF UGA selenocysteine codon and $75 \%$ at the mammalian deiodinase internal UGA codon indicate that recoding has variable efficiency (Suppmann et al., 1999; McCaughan et al., 1995). Apart from nucleotide context, the structured SECIS elements in both eukaryote and prokaryote systems are essential for UGA recoding. In prokaryotes, SECIS elements do not act simply to increase the local concentration of

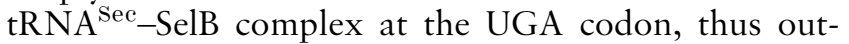
competing RF2; rather the SECIS element is required for delivery of the $\mathrm{tRNA}^{\mathrm{Sec}}-\mathrm{SelB}-\mathrm{GTP}$ ternary complex to the ribosome, and overexpressing the SelB-tRNA combination does not increase the efficiency of incorporation (Suppmann et al., 1999). This delivery mechanism, together with context effects, seems to be sufficient to much reduce alternative termination reactions. It seems likely the eukaryote SECIS elements function in the same way, although the mechanism used to achieve tRNA ${ }^{\mathrm{Sec}}$ UGA decoding when the SECIS element is located distally in the 3' UTR has not been established.

\section{Modulating termination efficiency using release factor concentration: eRF3 and [PSI]}

Programmed stop codon readthrough is principally employed by viruses and retrotransposons to control gene expression, and although some cellular gene examples are known, all seem to utilize cis mRNA elements such as stop codon context and downstream secondary structures. It is also possible to direct stop codon readthrough by lowering the cellular concentration of release factor (Stansfield et al., 1996). Intriguingly, in $S$. cerevisiae, lowered available concentrations of yeast eRF3 (the product of the SUP35 gene, Sup 35p) can exist naturally, since the yeast GTPase release factor eRF3 has prion properties. It can thus exist in alternative states, soluble or aggregated, known as $\left[\mathrm{psi}^{-}\right]$or $\left[\mathrm{PSI}^{+}\right]$, respectively (Wickner, 1994). A $\left[\mathrm{PSI}^{+}\right]$ state causes an enhanced nonsense suppression phenotype and thus readthough of natural stop codons at a certain frequency. Such readthrough events will Cterminally lengthen a small proportion of proteins, perhaps altering their turnover, localization or function. However, it is at present unknown whether a $\left[\mathrm{PSI}^{+}\right]$ state enhances the readthrough of the stop codon of any cellular gene sufficient to produce a detectable phenotype. However, $\left[\mathrm{PSI}^{+}\right]$-specific phenotypes other than nonsense suppression are known, and could possibly arise through such a C-terminal extension mechanism, discussed below (Eaglestone et al., 1999).

eRF3 (Sup35p) in the $\left[\mathrm{PSI}^{+}\right]$, aggregated state exists predominantly as cytoplasmic, high-molecular-mass oligomers, causing a weak nonsense suppressor pheno- type. Release factor eRF1 (Paushkin et al., 1996) and Upf1p, a component of the mRNA surveillance complex which regulates nonsense-mediated decay, are both proteins which bind eRF3, and both are found incorporated into $[P S I]$ eRF3 aggregates (Czaplinski et al., 1998).

Inheritance of $\left[\mathrm{PSI}^{+}\right]$eRF3 occurs by cytoplasmic mixing, primarily through yeast mating, and results in the conversion of soluble eRF3 (Sup35p) into the aggregated $\left[\mathrm{PSI}^{+}\right]$form. The propagation of $[\mathrm{PSI}]$ is dependent upon protein-protein interaction, and shows a spore (meiotic product) inheritance pattern of $4: 0$ $\left[P S I^{+}\right]:\left[p s i^{-}\right]$, characteristic of cytoplasmic, rather than nuclear, inheritance (Cox, 1965). The eRF3 $\left[\mathrm{PSI}^{+}\right]$prion exhibits many similarities to mammalian $\operatorname{PrP}$ prions, best illustrated by experiments to recreate de novo eRF3 (Sup35p) prion generation in vitro. Highly ordered amyloid-like fibres can be induced to form in vitro with purified eRF3, and fragments comprising the eRF3 Nterminal and middle domains (NM) or the N-terminal domain alone (Fig. 1; Glover et al., 1997). $\left[\mathrm{PSI}^{+}\right]$-form eRF3 fibres bind Congo red, are rich in $\beta$-sheet and can adopt different conformations, which are maintained throughout the fibre once self-perpetuation is initiated (Glover et al., 1997).

The eRF3 (Sup35p) protein is 685 amino acids in length and consists of three domains, each with different functions and properties (Fig. 1). The essential Cterminal domain of eRF3 is required for interaction with eRF1 in S. cerevisiae and Schizosaccharomyces pombe (Paushkin et al., 1997a). A second eRF1-binding site may be present on eRF3 in S. cerevisiae, spanning the Nterminal domain and middle $(\mathrm{M})$ domain of the release factor (Paushkin et al., 1997a). As of yet, no designated function has been ascribed to the middle domain of eRF3.

The N-terminal domain, comprising the first 114 amino acid residues, contains four glutamine-rich degenerate nonapeptide repeats, and is not required for release factor activity (Teravanesyan et al., 1993). In spite of this, the domain has been conserved in all eukaryotic eRF3 proteins, although not all $\mathrm{N}$-domains contain defined nonapeptide repeats. A number of lines of evidence indicate that the N-domain, with its peptide repeats, is centrally involved in $[P S I]$ inheritance: (i) overexpression of eRF3 (Sup35p) fragments comprising either the $\mathrm{N}$ - and $\mathrm{M}$-domains or $\mathrm{N}$-terminal domain of eRF3 alone can induce a $\left[\mathrm{PSI}^{+}\right]$state de novo (Paushkin et al., 1997a); (ii) single amino acid substitutions in one of the $\mathrm{N}$-domain nonapeptide repeats results in mutant eRF3 (Sup35p) unable to propagate or support the $\left[\mathrm{PSI}^{+}\right]$state (Doel et al., 1994; DePace et al., 1998); (iii) increasing or decreasing the number of nonapeptide repeats in the eRF3 $\mathrm{N}$-domain markedly increases or decreases, respectively, the spontaneous generation of the $\left[\mathrm{PSI}^{+}\right]$state in a $\left[\right.$psi $\left.{ }^{-}\right]$yeast (Liu \& Lindquist, 1999); and finally (iv) the fusion of SUP35 NM domains to GFP in a $\left[\mathrm{PSI}^{+}\right]$genetic background results in GFP aggregation in vivo (Patino et al., 1996). 


\section{Environmental and genetic factors influence the loss and reacquisition of $[P S I]$}

The ease with which aggregated $\left[\mathrm{PSI}^{+}\right] \mathrm{eRF} 3$ is inherited is mirrored by the efficiency with which a $\left[\mathrm{PSI}^{+}\right]$yeast strain can be converted to $\left[p s i^{-}\right]$. Known as curing, growth of a $\left[\mathrm{PSI}^{+}\right]$yeast in the presence of specific chemicals, such as guanidine hydrochloride $(5 \mathrm{mM})$ or glycerol (2 M) can liberate soluble eRF3 (Sup35p) from pre-established $\left[\mathrm{PSI}^{+}\right]$aggregates, and hence produce an isogenic yeast which is $\left[\right.$ psi $\left.^{-}\right]$(Tuite et al., 1981). A $\left[\mathrm{PSI}^{+}\right]$state can be re-established by eRF3 overexpression (Chernoff et al., 1993), although this phenomenon is strain dependent. Overexpression of the eRF3 NM domain is much more efficient at re-establishing a $\left[\mathrm{PSI}^{+}\right]$state (Paushkin et al., 1997b).

Proteins that adopt aberrant conformations can be disaggregated in vivo by the activity of molecular chaperones (Parsell et al., 1994), and the activity of the heat-shock 'disaggregase' protein Hsp104p can also play a role in the process of $[P S I]$ curing, evidence that $\left[\mathrm{PSI}^{+}\right]$defines an altered aggregation state of eRF3 (Chernoff et al., 1995; Schirmer \& Lindquist, 1997; Newnam et al., 1999). Both overexpression and disruption of the HSP104 gene result in the loss of [PSI] (Chernoff et al., 1995). Following Hsp104p overexpression, the $\left[\mathrm{PSI}^{+}\right]$state can be re-established by eRF3 (Sup35p) overexpression.

In addition to nonsense suppression itself, other discernible $\left[\mathrm{PSI}^{+}\right]$phenotypes, that of resistance to extreme temperatures and raised ethanol concentrations, have been detected (Eaglestone et al., 1999). Interestingly, the level of $\left[\mathrm{PSI}^{+}\right]$-mediated nonsense suppression was reduced when the $\left[\mathrm{PSI}^{+}\right]$strains were exposed to raised ethanol concentrations. Stress imposition thus produced transient reductions in the degree of eRF3 (Sup35p) aggregation in a $\left[\mathrm{PSI}^{+}\right]$strain, and thereby reduced nonsense suppression, without curing the $\left[\mathrm{PSI}^{+}\right]$state (Eaglestone et al., 1999). The reduced suppression phenotype observed was reversible, and a $\left[\mathrm{PSI}^{+}\right]$state restored upon ethanol removal. Such reversible control of nonsense suppression frequencies may indicate that under certain stress conditions $S$. cerevisiae may use the $\left[\mathrm{PSI}^{+}\right]$state to control readthrough of a natural stop codon(s) with the consequent C-terminal extension of the polypeptide. However, no ORFs subject to [PSI]programmed readthrough have yet been identified.

\section{tRNA suppressors of nonsense codons}

The failure of a release factor to recognize a stop codon normally triggers a default, alternative elongation event mediated by a suppressor tRNA. Stop codon recognition by the RF and termination competes directly with suppressor tRNAs, which can be cognate or mis-cognate for the termination codon (Fig. 3). Their affinity for the stop codon and A-site termination environment, relative to that of the RF, determines the stop codon readthrough frequency. Suppressors can be wild-type tRNAs which are miscognate for stop codons, and wobble base pair with the stop codon at the third codon position, or

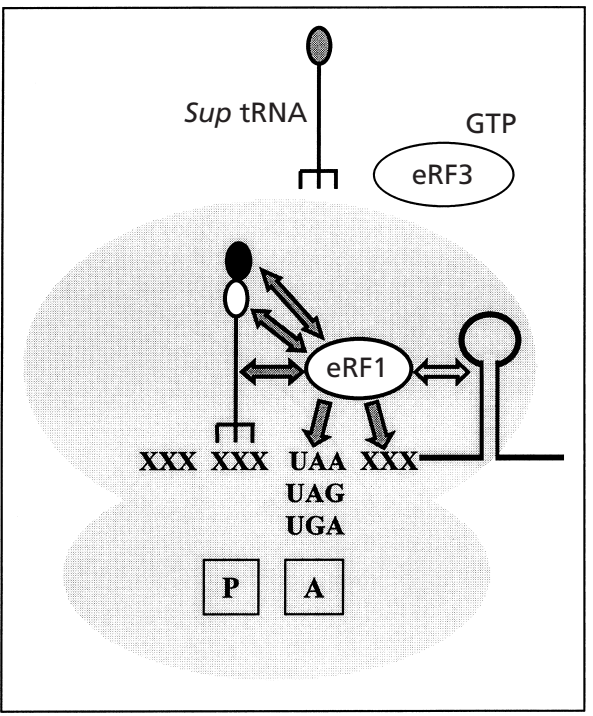

Fig. 3. Schematic representation of factors determining the efficiency of stop codon recognition by the class 1 release factors eRF1, RF1 and RF2. Cis-acting factors which influence termination efficiency include the nucleotides immediately downstream of the stop codon, particularly at the +1 and +3 positions, the stop codon itself, the P-site tRNA, and the ultimate and penultimate amino acids in the nascent polypeptide chain (dark grey arrows). The relative importance of these factors varies between eukaryotes and prokaryotes (see text for details). Other important cis factors, particularly in viral programmed stop codon readthrough signals, are downstream secondary structures, such as stem-loops and pseudoknots (light grey arrow). These may have indirect, as opposed to direct stearic or stereochemical effects on RF-stop codon interaction. Trans factors modulating competitive advantage of the RF over suppressor tRNAs include firstly the tRNAs themselves, which may be cognate or miscognate for stop codons, and secondly the abundance of the class 2 GTPase release factor eRF3.

cognate for a stop codon, either through mutation, or because in a given non-universal genetic code organism a stop codon has been reassigned to sense (discussed previously). A final category contains tRNAs which can be defined as P-site effectors of nonsense suppression. These tRNAs modulate A-site decoding events either through antagonism of release factor binding, or promotion of suppressor tRNA binding; in doing so, they contribute to the $5^{\prime}$ context effects on termination efficiency. Natural suppressor tRNAs will be considered in more detail below.

\section{Sense codon-cognate suppressor tRNAs}

Ochre (UAA) mutations can be suppressed in yeast in vivo by increasing the copy number of a wild-type CAAdecoding tRNA $\mathrm{U}^{\text {Gln }} \mathrm{UG}$ (where $\mathrm{U}^{*}$ represents modified uridine) (Pure et al., 1985). A second yeast CAGdecoding tRNA $\mathrm{CU}$ Gin , can suppress amber UAG codons when expressed on a multi-copy plasmid (Weiss \& Friedberg, 1986). In both these cases, UAA and UAG suppression requires a first anticodon position $G:: U$ wobble base pairing (Table 2). In another study, peptide 
sequencing identified amino acids inserted at the position of a premature UAG stop codon of a nonsense yeast STE6 allele. This stop codon, found in a nucleotide context unfavourable for eRF1 recognition, was suppressed by tyrosine, lysine and tryptophan tRNAs, although not by a tRNA ${ }^{\text {Gln }}$ as expected (Fearon et al., 1994).

Many plant viruses, together with some animal viruses, rely for propagation on programmed stop codon readthrough events, generally of stop codons in nucleotide contexts unfavourable for eRF recognition. This ensures correct relative proportions of structural and enzymic virus translation products. This has stimulated the search for the naturally suppressing tRNAs responsible for the stop codon readthrough. In plants, a wheat cytoplasmic tRNA $\mathrm{U}_{\mathrm{U}^{*} \mathrm{CG}}^{\mathrm{Arg}}$ has been purified with in vitro UGA suppressor activity, a decoding event requiring a first codon position $\mathrm{G}: \mathrm{U}$ wobble pairing (Baum \& Beier, 1998). A UGA stop codon in various virus programmed readthrough contexts was also efficiently suppressed in vitro by a plant tRNA $\mathrm{CrmCA}_{\mathrm{C}}^{\mathrm{Tr}}$, involving a $\mathrm{C}$ : A mispairing at the third codon position (Urban et al., 1996). A third plant tRNA, a tRNA $\mathrm{GCA}_{\mathrm{CAs}}^{\mathrm{Cys}}$ from tobacco, was also able to decode UGA in either the TMV or tobacco rattle virus (TRV) leaky contexts (Urban \& Beier, 1995). Finally, a plant tRNA $\mathrm{G}_{\mathrm{G} \psi \mathrm{A}}^{\mathrm{Ty}}$ (where $\psi$ represents pseudouridine) has been isolated that has both UAA and UAG suppression activity in vitro (Beier et al., 1984). Decoding of UAA and UAG requires noncanonical $G: A$ or $G: G$ pairings at the third codon position, respectively.

In mammalian cells, the identity of natural suppressor tRNAs involved in programmed readthrough of the Mo-MuLV) has been inferred by identifying the amino acid inserted at the position corresponding to the suppressed stop codon. This revealed that both UAG and UAA can direct glutamine incorporation as well as termination events, while UGA directs arginine, cysteine and tryptophan insertion (Feng et al., 1990).

Clearly, similar isoacceptor tRNA species act as natural tRNA suppressors in different systems studied via first or third codon position wobble base pairing. In all cases, however, nonsense suppression frequency directed by these miscognate tRNAs is only detectable when the stop codon is placed in a nucleotide context unfavourable for eRF binding. Clearly the structural properties of the suppressor tRNAs themselves will contribute to their mis-decoding of stop codons, as well as their nucleoside modifications, which are known to affect nonsense suppression efficiency in yeast and other systems (Dihanich et al., 1987).

\section{Post-termination ribosomal fates}

Following termination in both prokaryotes and eukaryotes, ribosomes must be released from the mRNA to take part in new rounds of translation initiation, a recycling activity which resides in specific protein factors. However, at least in eukaryote cells, the activity of such factors may be regulated; during the translation of certain small, upstream ORFs (uORFs), ribosomes may not be immediately released following termination, but may instead resume scanning. Eukaryote ribosomes which have resumed scanning can subsequently reinitiate at downstream AUG codons, or alternatively can play a role in complex pathways controlling mRNA stability, which are only now being slowly unravelled. These 'recycling alternative' events constitute novel forms of regulation of gene expression at a posttranscriptional level.

\section{Ribosomes are released from the MRNA and recycled post-termination}

Following termination, cells must recycle ribosomes, release factors and tRNAs to allow new rounds of protein synthesis. In prokaryotes, post-termination ribosome release requires the activity of the essential ribosome recycling factor (RRF; Janosi et al., 1998). A post-termination ribosomal complex becomes a preinitiation complex via a two-step mechanism. Firstly, RRF and GTP-complexed EF-G together split the postpeptidyl-release ribosome into its component subunits. In the second step, the initiation factor IF3 acts to remove the deacylated tRNA from the ribosomal P-site (Freistroffer et al., 1997; Pavlov et al., 1997; Karimi et al., 1999). In vivo, following termination and in the absence of RRF, prokaryote $70 \mathrm{~S}$ ribosomes can remain on the mRNA and continue to 'slide' downstream, and eventually reinitiate protein synthesis at any codon between 17 and $45 \mathrm{nt}$ downstream in a frame-independent manner (Janosi et al., 1998). In addition, RRF acting with EF-G can also reduce frameshift errors in vitro, and reduce missense translation error during peptide elongation (Janosi et al., 1996), although the mechanism of this activity is not well understood. The recently solved crystal structure of RRF from Thermotoga maritima has revealed that this protein, like eRF1, is a tRNA mimic, although a much more exact mimic than is eRF1 (Fig. 2; Selmer et al., 1999). RRF-tRNA mimicry is almost certainly a central feature of its activity in dissociating ribosomes. It has been proposed that following termination and peptidyl release, RRF binds to the A-site and is translocated by EF-G in the same way as peptidyl-tRNA is translocated. P-site occupancy by RRF is then the trigger for ribosomal dissociation (Selmer et al., 1999). This model has certain features which distinguish it from the proposal that IF3 acts to remove deacylated tRNA from the P-site following RRF-catalysed ribosome dissociation from the mRNA (Karimi et al., 1999). However, it might be that in bacteria, post-termination mechanisms differ dependent upon the ribosome proximity to a ribosomebinding site (Selmer et al., 1999).

The analogous ribosome recycling step in eukaryotes is in contrast completely uncharacterized; a homologous nucleus-encoded gene encoding an RRF-like protein is found in eukaryotes, but appears to be an organelle protein; in yeast, the $R R F 1$ gene has a mitochondrial targeting sequence, and disruption of the gene is nonlethal, generating a petite phenotype typical of disrupted 
mitochondrial function (I. Stansfield \& M. F. Tuite, unpublished). While other eukaryote RRF-encoding genes have been identified, they are all targeted to organelles such as chloroplasts (Rolland et al., 1999). No equivalent cytoplasmic form has yet been identified. One recent suggestion is that eRF3 may represent a combined RF3 and RRF activity, thus representing a eukaryote recycling function, although hard evidence to support this is lacking at present (Buckingham et al., 1997).

\section{Alternative post-termination events: resumed scanning}

In eukaryotes, post-termination ribosomes can experience different fates, regulated by the nucleotide context of the stop codon. This is best exemplified by the posttranscriptional regulation mechanisms of the S. cerevisiae GCN4 gene, which encodes a transcription factor involved in responses to amino acid starvation. The GCN4 mRNA 5' UTR contains four uORFs, of which only uORF1 and uORF4 are required for wild-type translational control of GCN4 expression (Mueller \& Hinnebusch, 1986). The precise details of this mechanism, and its relation to amino acid starvation responses, are reviewed elsewhere (Hinnebusch, 1997). Of importance to this discussion of regulation of (post-) termination events are the findings that ribosomes can resume scanning after terminating translation at uORF1, and that they can subsequently acquire competence to reinitiate at downstream AUG codons (Hinnebusch, 1997). In contrast, ribosomes terminating translation at the uORF4 termination codon are released from the mRNA (Abastado et al., 1991; Dever et al., 1992). This key difference between the behaviour of post-termination ribosomes at uORFs 1 and 4 results from the character of the nucleotide context immediately preceding ( $3 \mathrm{nt})$ and following (10 nt) the respective uORF stop codons (Grant \& Hinnebusch, 1994). An AU-rich nucleotide bias around the uORF1 stop codon triggers resumed scanning, whereas the GC-rich character of the corresponding uORF4 nucleotides promotes ribosome release and recycling. While the mechanism of action of these cis sequences is not fully understood, it is clear that no single sequence confers these properties, and that a wide variety of contexts can achieve the same effect (Grant \& Hinnebusch, 1994). uORFs in the 5' UTRs of the YAP1 and YAP2 genes, while having different sequences surrounding their stop codons, nevertheless have very similar properties to GCN4 uORFs 1 and 4, respectively, again an indication that primary sequence itself is not the prime determinant of the ability to promote resumed scanning (Vilela et al., 1998). It has been suggested that the GC-rich environment around the uORF4 stop codon may pause a terminating ribosome (perhaps via rRNA interaction) long enough to allow a putative recycling factor to bind, an event prevented by rapid termination at uORF1 (Grant \& Hinnebusch, 1994). The region 5' of uORF1 is also important for the reinitiation stimulating ability of uORF1, although this mRNA region lies outside that which would be occluded by a ribosome terminating at uORF1 (Grant et al., 1995).

In prokaryotic systems, small ORFs have peculiar properties, first highlighted by studies of the $\lambda$ bar minigenes, which encode dipeptides. During translation of such mini-genes, translation termination does not always take place, and in some instances, peptidyltRNA is released, stimulated by RRF, RF3 and the elongation factor EF-G; this reaction is detrimental to peptidyl hydrolase mutants expressing a mini-gene (peptidyl hydrolase breaks the tRNA nascent peptide bond in peptidyl tRNAs inappropriately released from the ribosome; Heurgue-Hamard et al., 1998). Translation initiation factors IF1 and IF2 also play a role in stimulating release of nascent peptidyl-tRNA without a prior termination reaction during short ORF (minigene) translation in vitro, and in vivo, overexpression of IF1 and IF2 cause growth defects in a peptidyl hydrolase mutant (Karimi et al., 1998). It is unclear at present whether these prokaryote termination-alternative reactions stimulated by mini-genes or uORFs have any significance for the regulatory properties conferred by uORF1 in the yeast GCN4 5' UTR. Certainly there is no direct evidence that translation termination actually takes place at either uORF 1 or uORF4, and the possibility that termination-alternative events take place has not been excluded.

\section{Conclusion and perspectives}

A skeleton outline of the mechanism of translation termination is now emerging from recent research. Arguably, two of the most important advances have been the solution of the eRF1 and RRF crystal structures, revealing their tRNA-like shapes and dimensions (Selmer et al., 1999; Song et al., 2000). The eRF1 structure solution now allows stop codon recognition, and eRF3-eRF1 and eRF1-ribosome interactions to be addressed in the context of structure (e.g. Bertram et al., 2000). However, despite similarities between eRFs and tRNAs, the detail of RF-stop codon interaction may be fundamentally different from that of tRNA anticodoncodon interaction; certainly the RF-stop codon recognition process is up to 60 times slower than sense codon decoding (Freistroffer et al., 2000). A recent model for eRF1 stop codon recognition also proposes that the stop codon bases may not be stacked as is probably the case in a sense codon interacting with cognate tRNA (Bertram et al., 2000). The involvement of nucleotides $3^{\prime}$ to the stop codon in class 1 RFs stop codon recognition also argues that stop codon recognition has unique features in comparison to sense codon decoding, which can now be addressed using the eRF1 structural information.

RF-tRNA mimcry also raises some intriguing questions, at least in prokaryote systems, where apparently an EF$\mathrm{Tu}$ like molecule is not required to bring the RF to the Asite (Freistroffer et al., 1997). What then provides the codon-'anticodon' fidelity checking mechanism which is fulfilled by EF-Tu when an aminoacyl tRNA is 
presented to the A-site? Termination at non-stop codons occurs at very low frequencies, so fidelity is obviously preserved by some mechanism (Freistroffer et al., 2000). It is also unclear how the release factors (which unlike tRNAs and EF-Tu/EF1- $\alpha$, are present in the cell at relatively low abundance) are selected quickly by the ribosome when a stop codon is located at the A-site.

The use of reconstituted translation systems using highly purified protein trans-acting factors has allowed an increasingly detailed picture to emerge, not just of termination itself, but also of the events which follow it. Eukaryote termination is less well defined; although in vitro termination reactions have been reconstituted, the precise role of the GTPase eRF3 is unclear, and our knowledge of how eukaryote release factors are removed from the ribosome, and the ribosomes subsequently removed from the mRNA, are hazy. The need to recycle ribosomes or subunits back to the $5^{\prime}$ end of the mRNA makes it likely that a specialized set of eukaryote factors exist for this purpose; either none have been identified to date, or factors already known have as yet undiscovered functions. The prospect of a fuller understanding emerging of the mechanism of both eukaryote and prokaryote termination in the near future is encouraging, providing a clear picture of how the last three codons in the genetic code are accurately decoded. It will also open the door to understanding how control of gene expression is exerted at the termination stage, and posttermination.

\section{Acknowledgements}

Work in the Stansfield laboratory is supported by funding from the Wellcome Trust and the Biotechnology and Biological Sciences Research Council.

\section{References}

Abastado, J. P., Miller, P. F., Jackson, B. M. \& Hinnebusch, A. G. (1991). Suppression of ribosomal reinitiation at upstream open reading frames in amino acid-starved cells forms the basis for GCN4 translational control. Mol Cell Biol 11, 486-496.

Al Karadaghi, S., Aevarsson, A., Garber, M., Zheltonosova, J. \& Liljas, A. (1996). The structure of elongation factor $G$ in complex with GDP: conformational flexibility and nucleotide exchange. Structure 4, 555-565.

Baum, M. \& Beier, H. (1998). Wheat cytoplasmic arginine tRNA isoacceptor with a $U^{*} \mathrm{CG}$ anticodon is an efficient UGA suppressor in vitro. Nucleic Acids Res 26, 1390-1395.

Beier, H., Barciszewska, M., Krupp, G., Mitnacht, R. \& Gross, H. J. (1984). UAG readthrough during TMV RNA translation: isolation and sequence of two tRNAs ${ }^{\text {Tyr }}$ with suppressor activity from tobacco plants. EMBO J 3, 351-356.

Berry, M. J., Banu, L., Chen, Y. Y., Mandel, S. J., Kieffer, J. D., Harney, J. W. \& Larsen, P. R. (1991). Recognition of UGA as a selenocysteine codon in type I deiodinase requires sequences in the 3' untranslated region. Nature 353, 273-276.

Berry, M. J., Banu, L., Harney, J. W. \& Larsen, P. R. (1993). Functional characterization of the eukaryotic SECIS elements which direct selenocysteine insertion at UGA codons. EMBO J 12, 3315-3322.

Bertram, G., Bell, H. A., Ritchie, D. W., Fullerton, G. \& Stansfield,
I. (2000). Terminating eukaryote translation: domain 1 of release factor eRF1 functions in stop codon recognition. RNA $\mathbf{6}$, 1236-1247.

Bjornsson, A., Mottagui-Tabar, S. \& Isaksson, L. A. (1996). Structure of the C-terminal end of the nascent peptide influences translation termination. EMBO J 15, 1696-1704.

Bock, A., Forchhammer, K., Heider, J. \& Baron, C. (1991). Selenoprotein synthesis: an expansion of the genetic code. Trends Biochem Sci 16, 463-467.

Bonetti, B., Fu, L. W., Moon, J. \& Bedwell, D. M. (1995). The efficiency of translation termination is determined by a synergistic interplay between upstream and downstream sequences in Saccharomyces cerevisiae. J Mol Biol 251, 334-345.

Brierley, I., Digard, P. \& Inglis, S. C. (1989). Characterization of an efficient coronavirus ribosomal frameshifting signal: requirement for an RNA pseudoknot. Cell 57, 537-547.

Brown, C. M. \& Tate, W. P. (1994). Direct recognition of mRNA stop signals by Escherichia coli polypeptide chain release factor two. J Biol Chem 269, 33164-33170.

Brown, C. M., Dinesh-Kumar, S. P. \& Miller, W. A. (1996). Local and distant sequences are required for efficient readthrough of the barley yellow dwarf virus PAV coat protein gene stop codon. $J$ Virol 70, 5884-5892.

Buckingham, R. H., Grentzmann, G. \& Kisselev, L. (1997). Polypeptide chain release factors. Mol Microbiol 24, 449-456.

Caron, F. \& Meyer, E. (1985). Does Paramecium primaurelia use a different genetic code in its macronucleus? Nature 314, 185-188.

Caskey, T., Forrester, W. C., Tate, W. P. \& Ward, C. D. (1984). Cloning of the Escherichia coli release factor 2 gene. J Bacteriol 158, 365-368.

Chernoff, Y. O., Derkach, I. L. \& Inge-Vechtomov, S. G. (1993). Multicopy SUP35 gene induces de-novo appearance of psi-like factors in the yeast Saccharomyces cerevisiae. Curr Genet 24, 268-270.

Chernoff, Y. O., Lindquist, S. L., Ono, B., Inge-Vechtomov, S. G. \& Liebman, S. W. (1995). Role of the chaperone protein Hsp104 in propagation of the yeast prion-like factor $[\operatorname{PSI}(+)]$. Science 268, 880-884.

Clare, J. J., Belcourt, M. \& Farabaugh, P. J. (1988). Efficient translational frameshifting occurs within a conserved sequence of the overlap between the two genes of a yeast Ty1 transposon. Proc Natl Acad Sci US A 85, 6816-6820.

Cox, B. S. (1965). $\Psi$, a cytoplasmic suppressor of super suppressor in yeast. Heredity 20, 505-521.

Craigen, W. J., Lee, C. C. \& Caskey, C. T. (1990). Recent advances in peptide chain termination. Mol Microbiol 4, 861-865.

Crawford, D.-J. G., Ito, K., Nakamura, Y. \& Tate, W. P. (1999). Indirect regulation of translational termination efficiency at highly expressed genes and recoding sites by the factor recycling function of Escherichia coli release factor RF3. EMBO J 18, 727-732.

Czaplinski, K., Ruizechevarria, M. J., Paushkin, S. V., Han, X., Weng, Y. M., Perlick, H. A., Dietz, H. C., Ter-Avanesyan, M. D. \& Peltz, S. W. (1998). The surveillance complex interacts with the translation release factors to enhance termination and degrade aberrant mRNAs. Genes Dev 12, 1665-1677.

DePace, A. H., Santoso, A., Hillner, P. \& Weissman, J. S. (1998). A critical role for amino-terminal glutamine/asparagine repeats in the formation and propagation of a yeast prion. Cell 93, $1241-1252$.

Dever, T. E., Feng, L., Wek, R. C., Cigan, A. M., Donahue, T. F. \& Hinnebusch, A. G. (1992). Phosphorylation of initiation factor 2 
alpha by protein kinase GCN2 mediates gene-specific translational control of GCN4 in yeast. Cell 68, 585-596.

Dihanich, M. E., Najarian, D., Clark, R., Gillman, E. C., Martin, N. C. \& Hopper, A. K. (1987). Isolation and characterization of MOD5, a gene required for isopentenylation of cytoplasmic and mitochondrial tRNAs of Saccharomyces cerevisiae. Mol Cell Biol 7, 177-184.

Dinesh-Kumar, S. P., Brault, V. \& Miller, W. A. (1992). Precise mapping and in vitro translation of a trifunctional subgenomic RNA of barley yellow dwarf virus. Virology 187, 711-722.

Doel, S. M., Mccready, S. J., Nierras, C. R. \& Cox, B. S. (1994). The dominant PNM2(-) mutation which eliminates the $p$ si-factor of Saccharomyces cerevisiae is the result of a missense mutation in the SUP35 gene. Genetics 137, 659-670.

Drugeon, G., Jean-Jean, O., Frolova, L., Le Goff, X., Philippe, M., Kisselev, L. \& Haenni, A.-L. (1997). Eukaryotic release factor 1 (eRF1) abolishes readthrough and competes with suppressor tRNAs at all three termination codons in messenger RNA. Nucleic Acids Res 25, 2254-2258.

Eaglestone, S. S., Cox, B. S. \& Tuite, M. F. (1999). Translation termination efficiency can be regulated in Saccharomyces cerevisiae by environmental stress through a prion-mediated mechanism. EMBO J 18, 1974-1981.

Engelberg-Kulka, H. (1981). UGA suppression by normal tRNA Trp in Escherichia coli: codon context effects. Nucleic Acids Res 9, 983-991.

Eurwilaichitr, L., Graves, F. M., Stansfield, I. \& Tuite, M. F. (1999). The C-terminus of eRF1 defines a functionally important domain for translation termination in Saccharomyces cerevisiae. Mol Microbiol 32, 485-496.

Fearon, K., McClendon, V., Bonetti, B. \& Bedwell, D. M. (1994). Premature translation termination mutations are efficiently suppressed in a highly conserved region of yeast Ste6p, a member of the ATP-binding cassette (ABC) transporter family. J Biol Chem 269, 17802-17808.

Feng, Y.-X., Copeland, T. D., Oroszlan, S., Rein, A. \& Levin, J. G. (1990). Identification of amino acids inserted during suppression of UAA and UGA termination codons at the gag-pol junction of Moloney murine leukemia virus. Proc Natl Acad Sci US A 87, 8860-8863.

Feng, Y.-X., Yuan, H., Rein, A. \& Levin, J. G. (1992). Bipartite signal for read-through suppression in murine leukemia virus mRNA: an eight-nucleotide purine-rich sequence immediately downstream of the gag termination codon followed by an RNA pseudoknot. J Virol 66, 5127-5132.

Fraser, C. M., Gocayne, J. D., White, O., Adams, M., Clayton, R., Fleischmann, R. D. \& Bult, C. J. (1995). The minimal gene complement of Mycoplasma genitalium. Science 270, 397-403.

Freistroffer, D. V., Pavlov, M. Y., MacDougall, J., Buckingham, R. H. \& Ehrenberg, M. (1997). Release factor RF3 in E. coli accelerates the dissociation of release factors RF1 and RF2 from the ribosome in a GTP-dependent manner. EMBO $J \mathbf{1 6}$, $4126-4133$.

Freistroffer, D. V., Kwiatkowski, M., Buckingham, R. H. \& Ehrenberg, M. (2000). The accuracy of codon recognition by polypeptide release factors. Proc Natl Acad Sci USA 97, 2046-2051.

Frolova, L., Le Goff, X., Rasmussen, H. H. \& 9 other authors (1994). A highly conserved eukaryotic protein family possessing properties of polypeptide chain release factor. Nature 372, 701-703.

Frolova, L. Y., Tsivkovskii, R. Y., Sivolobova, G. F., Oparina, N. Y.,
Serpinsky, O. I., Blinov, V. M., Tatkov, S. I. \& Kisselev, L. L. (1999). Mutations in the highly conserved GGQ motif of class 1 polypeptide release factors abolish ability of human eRF1 to trigger peptidyl-tRNA hydrolysis. RNA 5, 1014-1020.

Gesteland, R. F. \& Atkins, J. F. (1996). Recoding: dynamic reprogramming of translation. Annu Rev Biochem 65, 741-768.

Glover, J. R., Kowal, A. S., Schirmer, E. C., Patino, M. M., Liu, J. J. \& Lindquist, S. (1997). Self-seeded fibers formed by Sup35, the protein determinant of $\left[\mathrm{PSI}^{+}\right]$, a heritable prion-like factor of $S$. cerevisiae. Cell 89, 811-819.

Goelet, P., Lomonossoff, G. P., Butler, P. J. G., Akam, M. E., Gait, M. J. \& Kam, J. (1982). Nucleotide sequence of tobacco mosaic virus RNA. Proc Natl Acad Sci U S A 79, 5818-5822.

Grant, C. M. \& Hinnebusch, A. G. (1994). Effect of sequence context at stop codons on efficiency of reinitiation in GCN4 translational control. Mol Cell Biol 14, 606-618.

Grant, C. M., Miller, P. F. \& Hinnebusch, A. G. (1995). Sequences $5^{\prime}$ of the first upstream open reading frame in GCN4 mRNA are required for efficient translational reinitiation. Nucleic Acids Res 23, 3980-3988.

Grentzmann, G., Brechemier-Baey, D., Heurgue, V., Mora, L. \& Buckingham, R. H. (1994). Localization and characterization of the gene encoding release factor RF3 in Escherichia coli. Proc Natl Acad Sci US A 91, 5848-5852.

Grentzmann, G., Brechemier-Baey, D., Heurgue-Hamard, V. \& Buckingham, R. H. (1995). Function of polypeptide-chain release factor RF3 in Escherichia coli-RF3 action in termination is predominantly at UGA-containing stop signals. J Biol Chem 270, 10595-10600.

Grimm, M., Brunen Nieweler, C., Junker, V., Heckmann, K. \& Beier, H. (1998). The hypotrichous ciliate Euplotes octocarinatus has only one type of tRNA(cys) with GCA anticodon encoded on a single macronuclear DNA molecule. Nucleic Acids Res 26, 4557-4565.

Guilley, H., Wipf-Scheibel, C., Richards, K., Lecoq, H. \& Jonard, G. (1994). Nucleotide sequence of cucurbit aphid-borne yellows luteovirus. Virology 202, 1012-1017.

Hanyu, N., Kuchino, Y., Nishimura, S. \& Beier, H. (1986). Dramatic events in ciliate evolution - alteration of UAA and UAG termination codons to glutamine codons due to anticodon mutations in two tetrahymena transfer-RNAs ${ }^{\mathrm{Gln}}$. EMBO J 5, 1307-1311.

Heurgue-Hamard, V., Karimi, R., Mora, L., MacDougall, J., Leboeuf, C., Grentzmann, G., Ehrenberg, M. \& Buckingham, R. H. (1998). Ribosome release factor RF4 and termination factor RF3 are involved in dissociation of peptidyl-tRNA from the ribosome. EMBO J 17, 808-816.

Hinnebusch, A. G. (1997). Translational regulation of yeast GCN4. A window on factors that control initiator-tRNA binding to the ribosome. J Biol Chem 272, 21661-21664.

Hoshino, S., Imai, M., Mizutani, M., Kikuchi, Y., Hanaoka, F., Ui, M. \& Katada, T. (1998). Molecular cloning of a novel member of the eukaryotic polypeptide chain-releasing factors (eRF). Its identification as eRF3 interacting with eRF1. J Biol Chem 273, 22254-22259.

Hoshino, S., Imai, M., Kobayashi, T., Uchida, N. \& Katada, T. (1999). The eukaryotic polypeptide chain releasing factor (eRF3/GSPT) carrying the translation termination signal to the 3'-poly(A) tail of mRNA. J Biol Chem 274, 16677-16680.

Inagaki, Y. \& Doolittle, W. F. (2000). Evolution of the eukaryote translation termination system: origins of release factors. $\mathrm{Mol}$ Biol Evol 17, 882-889.

Inamine, J. M., Ho, K.-C., Loechel, S. \& Hu, P.-C. (1990). Evidence 
that UGA is read as a tryptophan codon rather than as a stop codon by Mycoplasma pneumoniae, Mycoplasma genitalium and Mycoplasma gallisepticum. J Bacteriol 172, 504-506.

Ishikawa, M., Meshi, T., Motoyoshi, F., Takamatsu, N. \& Okada, Y. (1986). In vitro mutagenesis of the putative replicase genes of tobacco mosaic virus. Nucleic Acids Res 14, 8291-8305.

Ito, K., Ebihara, K., Uno, M. \& Nakamura, Y. (1996). Conserved motifs in prokaryotic and eukaryotic polypeptide release factors: tRNA-protein mimicry hypothesis. Proc Natl Acad Sci US A 93, 5443-5448.

Ito, K., Ebihara, K. \& Nakamura, Y. (1998a). The stretch of Cterminal acidic amino acids of translational release factor eRF1 is a primary binding site for eRF3 of fission yeast. RNA 4, 958-972.

Ito, K., Uno, M. \& Nakamura, Y. (1998b). Single amino acid substitution in prokaryote polypeptide release factor 2 permits it to terminate translation at all three stop codons. Proc Natl Acad Sci U S A 95, 8165-8169.

Ito, K., Uno, M. \& Nakamura, Y. (2000). A tripeptide 'anticodon' deciphers stop codons in messenger RNA. Nature 403, 680-684.

Jalajakumari, M. B., Thomas, C. J., Halter, R. \& Manning, P. A. (1989). Genes for biosynthesis and assembly of CS3 pili of CFA/II enterotoxigenic Escherichia coli: novel regulation of pilus production by bypassing an amber codon. Mol Microbiol 3, 1685-1695.

Janosi, L., Ricker, R. \& Kaji, A. (1996). Dual functions of ribosome recycling factor in protein biosynthesis: disassembling the termination complex and preventing translational errors. Biochimie 78, 959-969.

Janosi, L., Mottagui-Tabar, S., Isaksson, L. A. \& 7 other authors (1998). Evidence for in vivo ribosome recycling, the fourth step in protein biosynthesis. EMBO J 17, 1141-1151.

Karamyshev, A. L., Ito, K. \& Nakamura, Y. (1999). Polypeptide release factor eRF1 from Tetrahymena thermophila: cDNA cloning, purification and complex formation with yeast eRF3. FEBS Lett 457, 483-488.

Karimi, R., Pavlov, M. Y., Heurgue-Hamard, V., Buckingham, R. H. \& Ehrenberg, M. (1998). Initiation factors IF1 and IF2 synergistically remove peptidyl-tRNAs with short polypeptides from the p-site of translating Escherichia coli ribosomes. J Mol Biol 281, 241-252.

Karimi, R., Pavlov, M. Y., Buckingham, R. H. \& Ehrenberg, M. (1999). Novel roles for classical factors at the interface between translation termination and initiation. Mol Cell 3, 601-609.

Keeling, P. J. \& Doolittle, W. F. (1997). Widespread and ancient distribution of a noncanonical genetic code in diplomonads. Mol Biol Evol 14, 895-901.

Kromayer, M., Wilting, R., Tormay, P. \& Bock, A. (1996). Domain structure of the prokaryotic selenocysteine-specific elongation factor SelB. J Mol Biol 262, 413-420.

Kuchino, Y., Hanyu, N., Tashiro, F. \& Nishimura, S. (1985). Tetrabymena thermophila glutamine tRNA and its gene that corresponds to UAA termination codon. Proc Natl Acad Sci US A 82, 4758-4762.

Lang, A., Friemert, C. \& Gassen, H. G. (1989). On the role of the termination factor RF-2 and the 16S RNA in protein synthesis. Eur J Biochem 180, 547-554.

Legoff, X., Philippe, M. \& Jean-Jean, O. (1997). Overexpression of human release factor 1 alone has an antisuppressor effect in human cells. Mol Cell Biol 17, 3164-3172.

Leng, P., Klatte, D. H., Schumann, G., Boeke, J. D. \& Steck, T. L. (1998). Skipper, an LTR retrotransposon of Dictyostelium. Nucleic Acids Res 26, 2008-2015.
Liu, J. J. \& Lindquist, S. (1999). Oligopeptide-repeat expansions modulate 'protein-only' inheritance in yeast. Nature 400, 573-576.

McCaughan, K. K., Brown, C. M., Dalphin, M. E., Berry, M. J. \& Tate, W. P. (1995). Translational termination efficiency in mammals is influenced by the base following the stop codon. Proc Natl Acad Sci U S A 92, 5431-5435.

Major, L. L., Poole, E. S., Dalphin, M. E., Mannering, S. A. \& Tate, W. P. (1996). Is the in-frame termination signal of the Escherichia coli release factor- 2 frameshift site weakened by a particularly poor context? Nucleic Acids Res 24, 2673-2678.

Matthews, G. D., Goodwin, T. J. D., Butler, M. I., Berryman, T. A. \& Poulter, R. T. M. (1997). pCal, a highly unusual Ty1/copia retrotransposon from the pathogenic yeast Candida albicans. $J$ Bacteriol 179, 7118-7128.

Meyer, F., Schmidt, H. J., Plumper, E., Hasilik, A., Mersmann, G., Meyer, H. E., Engstrom, A. \& Heckmann, K. (1991). UGA is translated as cysteine in pheromone-3 of Euplotes octocarinatus. Proc Natl Acad Sci US A 88, 3758-3761.

Mikuni, O., Ito, K., Moffat, J., Matsumura, K., McCaughan, K., Nobukuni, T., Tate, W. P. \& Nakamura, Y. (1994). Identification of the $\operatorname{prfC}$ gene, which encodes peptide-chain-release factor 3 of Escherichia coli. Proc Natl Acad Sci U S A 91, 5798-5802.

Mottagui-Tabar, S. \& Isaksson, L. A. (1998). The influence of the $5^{\prime}$ codon context on translation termination in Bacillus subtilis and Escherichia coli is similar but different from Salmonella typhimurium. Gene 212, 189-196.

Mottagui-Tabar, S., Bjornsson, A. \& Isaksson, L. A. (1994). The second to last amino acid in the nascent peptide as a codon context determinant. EMBO J 13, 249-257.

Mottagui-Tabar, S., Tuite, M. F. \& Isaksson, L. A. (1998). The influence of $5^{\prime}$ codon context on translation termination in Saccharomyces cerevisiae. Eur J Biochem 257, 249-254.

Mueller, P. P. \& Hinnebusch, A. G. (1986). Multiple upstream AUG codons mediate translational control of GCN4. Cell 45, 201-207.

Newnam, G. P., Wegrzyn, R. D., Lindquist, S. L. \& Chernoff, Y. O. (1999). Antagonistic interactions between yeast chaperones hsp104 and hsp70 in prion curing. Mol Cell Biol 19, 1325-1333.

Nissen, P., Kjeldgaard, M., Thirup, S., Polekhina, G., Reshetnikova, L., Clark, B. F. \& Nyborg, J. (1995). Crystal structure of the ternary complex of Phe-tRNAPhe, EF-Tu, and a GTP analog. Science 270, 1464-1472.

Parsell, D. A., Kowal, A. S., Singer, M. A. \& Lindquist, S. (1994). Protein disaggregation mediated by heat shock protein Hsp104. Nature 372, 475-478.

Patino, M. M., Liu, J. J., Glover, J. R. \& Lindquist, S. (1996). Support for the prion hypothesis for inheritance of a phenotypic trait in yeast. Science 273, 622-626.

Paushkin, S. V., Kushnirov, V. V., Smirnov, V. N. \& TerAvanesyan, M. D. (1996). Propagation of the yeast prion-like $[p s i(+)]$ determinant is mediated by oligomerization of the Sup35-encoded polypeptide-chain release factor. EMBO J 15, 3127-3134.

Paushkin, S. V., Kushnirov, V. V., Smirnov, V. N. \& TerAvanesyan, M. D. (1997a). Interaction between yeast Sup45p (eRF1) and Sup35p (eRF3) polypeptide chain release factors: implications for prion-dependent regulation. Mol Cell Biol 17, 2798-2805.

Paushkin, S. V., Kushnirov, V. V., Smirnov, V. N. \& TerAvanesyan, M. D. (1997b). In vitro propagation of the prion-like state of yeast Sup35 protein. Science 277, 381-383. 
Pavlov, M. Y., Freistroffer, D. V., MacDougall, J., Buckingham, R. H. \& Ehrenberg, M. (1997). Fast recycling of Escherichia coli ribosomes requires both ribosome recycling factor (RRF) and release factor RF3. EMBO J 16, 4134-4141.

Pelham, H. R. B. (1978). Leaky UAG termination codon in tobacco mosaic virus RNA. Nature 272, 469-471.

Poole, E. S., Brown, C. M. \& Tate, W. P. (1995). The identity of the base following the stop codon determines the efficiency of in-vivo translational termination in Escherichia coli. EMBO $J$ 14, 151-158.

Poole, E. S., Major, L. L., Mannering, S. A. \& Tate, W. P. (1998). Translation termination in Escherichia coli: three bases following the stop codon crosslink to release factor 2 and affect the decoding efficiency of UGA-containing signals. Nucleic Acids Res 26, 954-960.

Pure, G. A., Robinson, G. W., Naumovski, L. \& Friedberg, E. C. (1985). Partial suppression of an ochre mutation in Saccharomyces cerevisiae by multicopy plasmids containing a normal yeast transfer RNA-Gln gene. J Mol Biol 183, 31-42.

Robertus, J. D., Ladner, J. E., Finch, J. T., Rhodes, D., Brown, R. S., Clark, B. F. \& Klug, A. (1974). Structure of yeast phenylalanine tRNA at 3 A resolution. Nature 250, 546-551.

Rolland, N., Janosi, L., Block, M. A. \& 7 other authors (1999). Plant ribosome recycling factor homologue is a chloroplastic protein and is bactericidal in Escherichia coli carrying temperaturesensitive ribosome recycling factor. Proc Natl Acad Sci US A 96, 5464-5469.

Schirmer, E. C. \& Lindquist, S. (1997). Interactions of the chaperone Hsp104 with yeast Sup35 and mammalian PrP. Proc Natl Acad Sci U S A 94, 13932-13937.

Scolnick, E., Tomkins, R., Caskey, T. \& Nirenberg, M. (1968). Release factors differing in specificity for terminator codons. Proc Natl Acad Sci US A 61, 768-774.

Selmer, M., Al Karadaghi, S., Hirokawa, G., Kaji, A. \& Liljas, A. (1999). Crystal structure of Thermotoga maritima ribosome recycling factor: a tRNA mimic. Science 286, 2349-2352.

Skuzeski, J. M. \& Atkins, J. F. (1990). Analysis of leaky viral translation termination codons in vivo by transient expression of improved beta-glucuronidase vectors. Plant Mol Biol 15, 65-79.

Skuzeski, J. M., Nichols, L. M., Gesteland, R. F. \& Atkins, J. F. (1991). The signal for a leaky UAG stop codon in several plant viruses includes the two downstream codons. J Mol Biol 218, 365-373.

Somogyi, P., Jenner, A. J., Brierley, I. \& Inglis, S. C. (1993). Ribosomal pausing during translation of an RNA pseudoknot. Mol Cell Biol 13, 6931-6940.

Song, H., Mugnier, P., Webb, H. M., Evans, D. R., Tuite, M. F., Hemmings, B. A. \& Barford, D. (2000). The crystal structure of human eukaryotic release factor eRF1 - mechanism of stop codon recognition and peptidyl-tRNA hydrolysis. Cell 100, 311-321.

Stansfield, I. \& Tuite, M. F. (1994). Polypeptide chain termination in Saccharomyces cerevisiae. Curr Genet 25, 385-395.

Stansfield, I., Jones, K. M., Kushnirov, V. V. \& 7 other authors (1995). The products of the SUP45 (eRF1) and SUP35 genes interact to mediate translation termination in Saccharomyces cerevisiae. EMBO J 14, 4365-4373.

Stansfield, I., Eurwilaichitr, L. \& Tuite, M. F. (1996). Depletion in the levels of the release factor eRF1 causes reduction in the efficiency of translation termination in yeast. Mol Microbiol 20, 1135-1143.

Suppmann, S., Persson, B. C. \& Bock, A. (1999). Dynamics and efficiency in vivo of UGA-directed selenocysteine insertion at the ribosome. EMBO J 18, 2284-2293.

Ter-Avanesyan, M. D., Kushnirov, V. V., Dagkesamanskaya, A. R., Didichenko, S. A., Chernoff, Y. O., Inge-Vechtomov, S. G. \& Smirnov, V. N. (1993). Deletion analysis of the SUP35 gene of the yeast Saccharomyces cerevisiae reveals two non-overlapping functional regions in the encoded protein. Mol Microbiol 7, 683-692.

Tuite, M. F., Mundy, C. R. \& Cox, B. S. (1981). Agents that cause a high frequency of genetic change from $[p s i+]$ to $[p s i-]$ in Saccharomyces cerevisiae. Genetics 98, 691-711.

Urban, C. \& Beier, H. (1995). Cysteine tRNAs of plant origin as novel UGA suppressors. Nucleic Acids Res 23, 4591-4597.

Urban, C., Zerfass, K., Fingerhut, C. \& Beier, H. (1996). UGA suppression by tRNACmCATrp occurs in diverse virus RNAs due to a limited influence of the codon context. Nucleic Acids Res 24, 3424-3430.

Vilela, C., Linz, B., Rodrigues-Pousada, C. \& McCarthy, J. E. G. (1998). The yeast transcription factor genes YAP1 and YAP2 are subject to differential control at the levels of both translation and mRNA stability. Nucleic Acids Res 26, 1150-1159.

Weiner, A. M. \& Weber, K. (1973). A single UGA codon functions as a natural termination signal in the coliphage $\mathrm{Q} \beta$ coat protein cistron. J Mol Biol 80, 837-855.

Weiss, R. B., Murphy, J. P. \& Gallant, J. A. (1984). Genetic screen for cloned release factor genes. J Bacteriol 158, 362-364.

Weiss, W. A. \& Friedberg, E. C. (1986). Normal yeast tranfer $\mathrm{RNA}_{\mathrm{CAG}}$ Gln can suppress amber codons and is encoded by an essential gene. J Mol Biol 192, 725-735.

Wickner, R. B. (1994). [URE3] as an altered URE2 protein: evidence for a prion analog in Saccharomyces cerevisiae. Science 264, 566-569.

Wills, N. M., Gesteland, R. F. \& Atkins, J. F. (1991). Evidence that a downstream pseudoknot is required for translational readthrough of the Moloney murine leukemia virus gag stop codon. Proc Natl Acad Sci U S A 88, 6991-6995.

Yamao, F., Muto, A., Kawauchi, Y., Iwami, M., Iwagami, S., Azumi, Y. \& Osawa, S. (1985) UGA is read as tryptophan in Mycoplasma capricolum. Proc Natl Acad Sci U S A 82, 2306-2309.

Yoshinaka, Y., Katoh, I., Copeland, T. D. \& Oroszlan, S. (1985). Murine leukemia virus protease is encoded by the gag-pol gene and is synthesized through suppression of an amber termination codon. Proc Natl Acad Sci U S A 82, 1618-1622.

Zhouravleva, G., Frolova, L., Le Goff, X., Le Guellec, R., IngeVechtomov, S., Kisselev, L. \& Philippe, M. (1995). Termination of translation in eukaryotes is governed by two interacting polypeptide chain release factors, eRF1 and eRF3. EMBO J 14, 4065-4072. 